\title{
Feminist Perspectives on Learning, Media and Technology: Recognition and Future Contributions
}

As I write this editorial the UK is celebrating the 100 year anniversary of the Representation of the People Act. This law, passed on the $6^{\text {th }}$ of February in 1991, allowed some women to vote for the first time. While imperfect (only women over the age of 35 who owned property or were married to someone who did could vote; compared to all men over the age of 21 regardless of home ownership status) it was an important historical moment for equality between men and women in the UK. It seems appropriate then, to use this editorial as recognition of the important work that has been carried out that uses a feminist lens to exploring issues of Ed Tech or Society and Technology more generally; and to encourage more authors working in the area of learning, media and technology to submit work that engages with feminist debates to the journal.

The different schools of feminist thought and ways of thinking about the relationships between gender and digitally-mediated life and learning highlight the need to make visible and challenge the ways that technologies can concretise, exacerbate and reconfigure inequalities between men and women in society. Scholars such as, Haraway, McRobbie, Turkle, Suchman and Wajcman, have all, in different ways, encouraged a more critical engagement with the way in which gender, ethnicity, class, sexuality and disability interrelate in the understanding of ourselves and the digital world; and are vital theoretical resources for those engaged in feminist research in studies of education and technology.

In an array of contexts and settings, there are concerns about the inequalities in the ways that men and women access, use, create and modify technology - leading to the risks of reinforcing or exacerbating existing socio-economic inequalities related to employment structures in the technology industries; and the lack of power women then have in shaping the future of society that is increasingly shaped by the digital (Ashcraft, 2015). Education and Technology are often seen as a way to try to address some of these inequalities, through for example, improving access to education, encouraging coding and tech interests in and outside school, and creating spaces where young people have a chance to make visible, critique and challenge these inequalities. Indeed, at present, there are a plethora of programmes globally, aimed at trying to get girls to code and engaged in the tech industry.

Of course, there are many challenges to this. Education and Technology need to be considered within the wider social context and are highly unlikely by themselves to address such complex structural problems, particularly where the tech industry is itself part of the problem. Furthermore, technology rarely straightforwardly mends problems of access to Education. Appropriate interventions are also challenging as, of course, technology is not neutral nor are 'girls' or 'women' one monolithic group. Yet, such interventions can make visible issues of power and representation, even if the outcomes are complex. Indeed, a number of the papers in a recent special issue of Learning, Media and Technology on youth media production (Dussel and Dahya, 2017) took a feminist perspective and highlighted this very topic. For example, Judge and Tuite drew attention to the relationships between gender, power, inequality in young people's digital media creation at university; highlighting the gendered qualities of the artefacts produced (Judge and Tuite, 2017). Through a poststructural analysis of gender and power relations, Fisher and Jensen provided into insight into the way such relationships are sometimes reconfigured in complex ways, as a result of 
feminist interventions where girls and boys were encouraged to reflect and critique gaming culture, and girls were supported in making their own digital games (Fisher and Jenson, 2017).

However, it is reasonable to suggest that there is a need for more research that applies a feminist lends in the Ed Tech field. There are continued problems with the ways that gender and other inequalities are encoded into the technologies we routinely use for learning and everyday life; and the power and representational imbalances in the Ed Tech and Technology industry more widely. Indeed, in research in this area, due to the common, typically instrumental characteristics of research around learning, media and technology, gender is often reduced simply to a 'neutral' variable in a model when trying to determine the educational impact or outcome.

A classic example of this would be the current excitement around the creation and use of personalised (learning) assistants, where many recurring themes can be identified. It is notable, for example, how Amazon's Echo (Alexa); Apple's (Siri); and Microsoft's (Cortana) were all initially designed with a female voice as the default option. This move was defended as merely a design orientated one - as it was suggested that men and women prefer female voices when instructing the assistant to carry out the (relatively menial) tasks that such a system is designed to fulfil. Yet, there are bigger questions to ask here about why this is the case; and indeed how these kinds of discussions of "preference" as if they were merely neutral facts and not reinforcing existing gender stereotypes can, and has, impacted the design of educational systems. Similarly, recent work on commercial visual classification systems highlighted how the classifiers that have been built by commercial companies tend to work best on male as opposed to female faces and perform better on lighter than darker skin, with the worst outcomes of all for the accuracy of classifying darker skinned females (Buolamwini and Gebru, 2018). Defenders of this outcome argue that this was merely an unintended effect due to a lack of images of females of varied skin colours to train the classifier on but this is not an acceptable (or indeed correct) argument.

Beyond gender being incorporated into design decisions, the rise of personalised learning systems tend to prioritise and support highly acquisition based forms of learning. They are most typically designed to offer the most 'efficient' form of 'knowledge transmission' and assume one correct answer. This set of beliefs about learning, teaching and education do not fit well with the core principles of feminist pedagogy and epistemology, and does not allow for debate, or to enable all parts of the curriculum to be properly explored, considered or connected to wider discussions of power and inequality in the Digital Society.

Technology for learning and Education does not need to be designed or thought of in this way. We would very much welcome those who take a feminist perspective to support discussion of alternative views, arguments, and perspectives of the future of Learning, Media and Technology to publish in the journal.

\section{References}

Ashcraft, C. (2015). Technology and sexuality - what's the connection? Addressing youth sexualities in efforts to increase girls' participation in computing. Learning, Media and Technology, 40(4), 437457 
Buolamwini, J and Gebru, T. (2018) Gender Shades: Intersectional Accuracy Disparities in Commercial Gender Classification. Proceedings of Machine Learning Research 81:1\{15, 2018 Conference on Fairness, Accountability, and Transparency

Dussel, I., \& Dahya, N. (2017). Introduction: problematizing voice and representation in youth media production. Learning, Media and Technology, 42(1), 1-7.

Fisher, S and Jenson, J. (2017) Producing alternative gender orders: a critical look at girls and gaming. Learning, Media and Technology, 42(1), 87-99.

Judge, M. and Tuite, D. (2017). Leaders or led? A qualitative analysis of how young people explore express and experiment via new media in an Irish higher education context. Learning, Media and Technology, 42(1), 28-53. 